\title{
A simple proof for the number of tilings of quartered Aztec diamonds
}

\author{
Tri Lai \\ Department of Mathematics \\ Indiana University \\ Bloomington, IN 47405, U.S.A. \\ tmlai@indiana.edu
}

Submitted: Jun 4, 2013; Accepted: Dec 30, 2013; Published: Jan 12, 2014

Mathematics Subject Classifications: 05A15, 05C70

\begin{abstract}
We get four quartered Aztec diamonds by dividing an Aztec diamond region by two zigzag cuts passing its center. W. Jockusch and J. Propp (in an unpublished work) found that the number of tilings of quartered Aztec diamonds is given by simple product formulas. In this paper we present a simple proof for this result.
\end{abstract}

Keywords: Aztec diamond, domino, tilings, perfect matchings

\section{Introduction}

In this paper a (lattice) region is a connected union of unit squares in the square lattice. A domino is the union of two unit squares that share an edge. A (domino) tiling of a region $R$ is a covering of $R$ by dominos such that there are no gaps or overlaps. Denote by $\mathrm{T}(R)$ the number of tilings of the region $R$.

The Aztec diamond of order $n$ is defined to be the union of all the unit squares with integral corners $(x, y)$ satisfying $|x|+|y| \leqslant n+1$. The Aztec diamond of order 8 is shown in Figure 1(a). In [3] it was shown that the number of tilings of the Aztec diamond of order $n$ is $2^{n(n+1)}$.

We are interested in three related families of regions first introduced by Jockusch and Propp [5] as follows. Divide the Aztec diamond of order $n$ into two congruent parts by a zigzag cut with 2 -unit steps (see Figure 1(b) for an example with $n=8$ ). By superimposing two such zigzag cuts that pass the center of the Aztec diamond we partition the region into four parts, called quartered Aztec diamonds. Up to symmetry, there are essentially two different ways we can superimpose the two cuts. For one of them, we obtained a fourfold rotational symmetric pattern, and four resulting parts are congruent. 


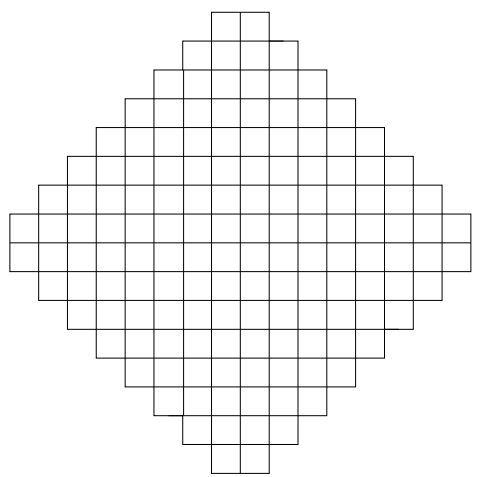

(a)

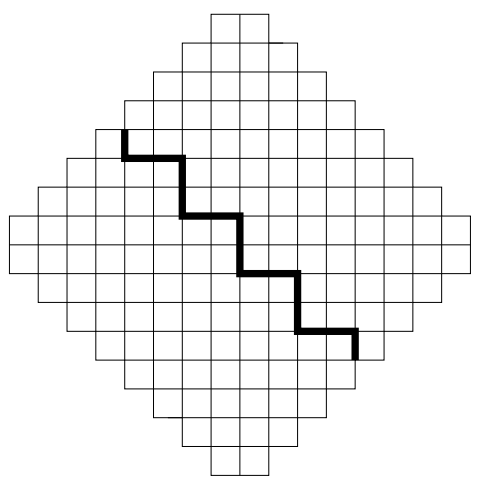

(b)

Figure 1: The Aztec diamond region of order 8, and its division into two congruent parts.

Denote by $R(n)$ these quartered Aztec diamonds (see Figure 2(a)). For the other, the obtained pattern has Klein 4-group reflection symmetry and there are two different kinds of quartered Aztec diamonds (see Figure 2 (b)); they are called abutting and non-abutting quartered Aztec diamonds. Denote by $K_{a}(n)$ and $K_{n a}(n)$ the abutting and non-abutting quartered Aztec diamonds of order $n$, respectively.

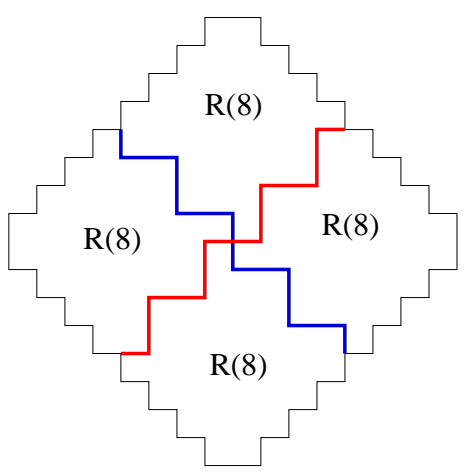

(a)

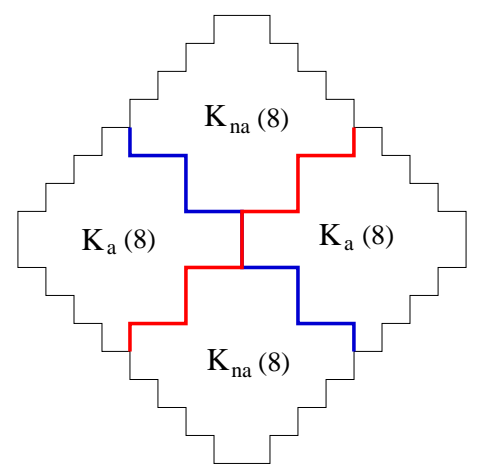

(b)

Figure 2: Three kinds of quartered Aztec diamonds of order 8.

The number of tilings of a quartered Aztec diamond is given by the theorem stated below.

Theorem 1 (Jockusch and Propp [5]). For any positive integer $n$

$$
\begin{gathered}
\mathrm{T}(R(4 n+1))=\mathrm{T}(R(4 n+2))=0, \\
\mathrm{~T}(R(4 n))=2^{n} \mathrm{~T}(R(4 n-1))=2^{n(3 n-1) / 2} \prod_{1 \leqslant i<j \leqslant n} \frac{2 i+2 j-1}{i+j-1}, \\
\mathrm{~T}\left(K_{a}(4 n-2)\right)=\mathrm{T}\left(K_{a}(4 n)\right)=2^{n(3 n-1) / 2} \prod_{1 \leqslant i<j \leqslant n} \frac{2 i+2 j-3}{i+j-1},
\end{gathered}
$$




$$
\begin{gathered}
\mathrm{T}\left(K_{a}(4 n-1)\right)=\mathrm{T}\left(K_{a}(4 n+1)\right)=2^{n(3 n-3) / 2} \prod_{1 \leqslant i \leqslant j \leqslant n} \frac{2 i+2 j-1}{i+j-1}, \\
\mathrm{~T}\left(K_{n a}(4 n)\right)=\mathrm{T}\left(K_{n a}(4 n+2)\right)=2^{n(3 n-1) / 2} \prod_{1 \leqslant i \leqslant j \leqslant n} \frac{2 i+2 j-1}{i+j-1}, \\
\mathrm{~T}\left(K_{n a}(4 n-3)\right)=\mathrm{T}\left(K_{n a}(4 n-1)\right)=2^{n(3 n-3) / 2} \prod_{1 \leqslant i<j \leqslant n} \frac{2 i+2 j-3}{i+j-1} .
\end{gathered}
$$

Juckusch and Propp [5] presented a proof for Theorem 1 by investigating properties of antisymmetric monotone triangles. In this paper, we prove Theorem 1 by a visual way using a certain factorization theorem due to Ciucu [1].

\section{Proof of Theorem 1}

We have 4 recurrences that were proved by Ciucu in [2], Theorem 4.1.

Lemma 2. For all $n \geqslant 1$

$$
\begin{gathered}
\mathrm{T}(R(4 n))=2^{n} \mathrm{~T}(R(4 n-1)), \\
\mathrm{T}\left(K_{n a}(4 n+1)\right)=2^{n} \mathrm{~T}\left(K_{n a}(4 n)\right), \\
\mathrm{T}\left(K_{n a}(4 n)\right)=2^{n} \mathrm{~T}\left(K_{a}(4 n-1)\right), \\
\mathrm{T}\left(K_{a}(4 n-2)\right)=2^{n} \mathrm{~T}\left(K_{n a}(4 n-3)\right) .
\end{gathered}
$$

A perfect matching of a graph $G$ is a collection of edges such that each vertex of $G$ is adjacent to exactly one selected edge. Denote by $\mathrm{M}(G)$ the number of perfect matchings of $G$.

The dual graph of a region $R$ (on the square lattice) is the graph whose vertices are unit square in $R$ and whose edges connect precisely two unit squares sharing an edge. By a well-known bijection between tilings of a region and perfect matchings of its dual graph, we enumerate perfect matchings of the dual graph of a region rather than enumerating its tilings directly. Since we are considering only regions in the square lattice, one can view the dual graphs of those regions as subgraphs of the infinite square grid $\mathbb{Z}^{2}$.

An edge in a graph $G$ is called a forced edge, if it is in every perfect matching of $G$. One can remove some forced edges and the vertices incident to them from a graph to get a new graph with the same number of perfect matchings. We have the following lemma by considering forced edges in the dual graphs of quartered Aztec diamonds.

Lemma 3. For any $n \geqslant 1$

$$
\begin{gathered}
\mathrm{T}\left(K_{a}(4 n-2)\right)=\mathrm{T}\left(K_{a}(4 n)\right), \\
\mathrm{T}\left(K_{a}(4 n-1)\right)=\mathrm{T}\left(K_{a}(4 n+1)\right), \\
\mathrm{T}\left(K_{n a}(4 n)\right)=\mathrm{T}\left(K_{n a}(4 n+2)\right), \\
\mathrm{T}\left(K_{n a}(4 n+1)\right)=\mathrm{T}\left(K_{n a}(4 n+3)\right) .
\end{gathered}
$$


Proof. Instead of comparing the numbers of tilings of the regions, we compare the numbers of perfect matchings of their dual graphs. In each of the four identities, the dual graph of the region on the left hand side is obtained from the dual graph of the region on the right hand side by removing forced edges and the vertices incident to them. The proofs of the identities (11)-(14) are illustrated by Figures 3 (a)-(d), respectively. In these figures, the forced edges are represented by the bold horizontal edges, and the dual graph of the region on the left hand side of each identity is represented by the graph consisting of shaded unit squares.

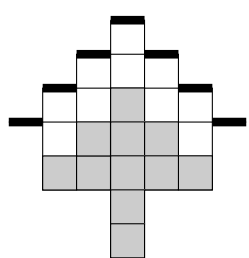

(a) Obtaining the dual graph of $\mathrm{K}_{\mathrm{a}}(6)$ from the dual graph of $\mathrm{K}_{\mathrm{a}}(8)$

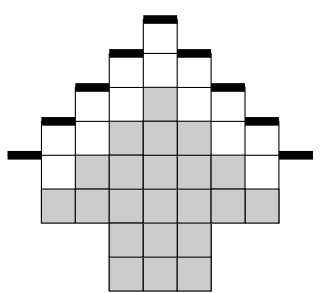

(c) Obtaining the dual graph of $\mathrm{K}_{\mathrm{na}}(8)$ from the dual graph of $\mathrm{K}_{\mathrm{na}}(10)$

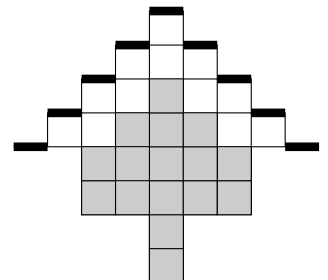

(b) Obtaining the dual graph of $\mathrm{K}_{\mathrm{a}}(7)$ from the dual graph of $\mathrm{K}_{\mathrm{a}}(9)$

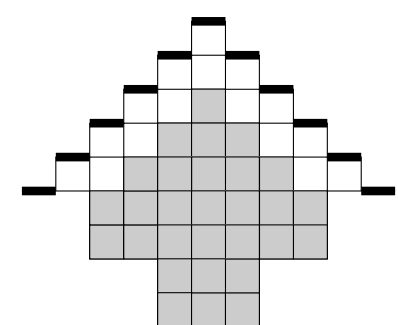

(d) Obtaining the dual graph of $\mathrm{K}_{\mathrm{na}}(9)$ from the dual graph of $\mathrm{K}_{\mathrm{na}}(11)$

Figure 3: Illustrating the proof of Lemma 3.

Next, we consider a well-known family of graphs as follows. Consider a $(2 m+1) \times$ $(2 n+1)$ rectangular chessboard and suppose that the corners are black. The $m \times n$ Aztec rectangle is the graph whose vertices are the white square and whose edges connect precisely those pairs of white squares that are diagonally adjacent (see Figure 4(a) for an example with $m=3$ and $n=5$ ). We are interested in the the number of perfect matchings of two families of holey Aztec rectangles as follows.

Lemma 4 (see [1], (4.4); or [6], Lemma 1). Denote by $A R_{m, n}\left(\left\{a_{1}, \ldots, a_{k}\right\}\right)$ the graph obtained from the $m \times n$ Aztec rectangle by removing all the vertices in the bottom-most row, except for the $a_{1}$-st, the $a_{2}-n d, \ldots$, and the $a_{m}$-th vertex (see Figure 4(b) for an example with $\left.m=3, n=5, a_{1}=1, a_{2}=3, a_{3}=5\right)$. Then

$$
\mathrm{M}\left(A R_{m, n}\left(\left\{a_{1}, \ldots, a_{k}\right\}\right)\right)=2^{m(m+1) / 2} \prod_{1 \leqslant i<j \leqslant m} \frac{a_{j}-a_{i}}{j-i} .
$$




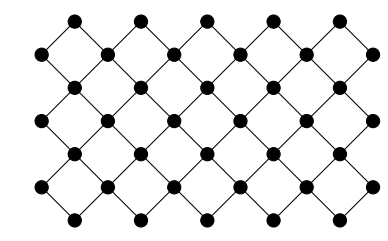

(a)

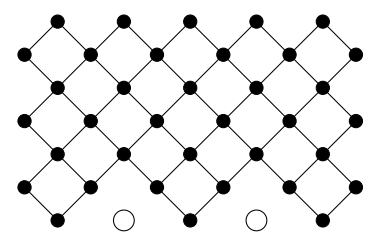

(b)

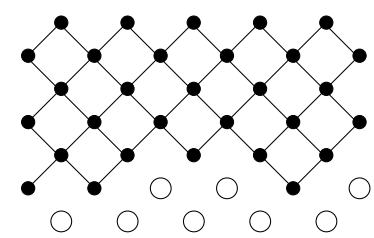

(c)

Figure 4: The Aztec rectangle and two holey Aztec rectangles of order $3 \times 5$. The white circles indicate the vertices removed.

Next, we consider a variant of the lemma above.

Lemma 5 (see [4], Lemma 2; or [6], Lemma 2). Denote by $\overline{A R}_{m, n}\left(\left\{a_{1}, \ldots, a_{k}\right\}\right)$ the graph obtained from the $m \times n$ Aztec rectangle by removing all the vertices in the bottom-most row, and removing the $a_{1}$-st, the $a_{2}-n d, \ldots$, and the $a_{m}$-th vertex in the next row (see Figure $4(c)$, for and example with $\left.m=3, n=5, a_{1}=3, a_{2}=4, a_{3}=6\right)$. Then

$$
\mathrm{M}\left(\overline{A R}_{m, n}\left(\left\{a_{1}, \ldots, a_{k}\right\}\right)\right)=2^{m(m-1) / 2} \prod_{1 \leqslant i<j \leqslant m} \frac{a_{j}-a_{i}}{j-i} .
$$

Next, we quote a useful result due to Ciucu [1]. However, we need some new definitions and terminologies before presenting the statement of the result.

Consider a weighted graph $G$ (i.e. a graph with a weight assignment on its edges). We define the weight of a perfect matching of $G$ to be the product of weights on its constituent edges. We define the operation $\mathrm{M}(G)$ in this case to be the sum of the weights of all perfect matchings of $G$. We call $\mathrm{M}(G)$ the matching generating function of $G$. We notice that if all edges of $G$ have weight 1, the matching generating function $\mathrm{M}(G)$ is exactly the number of perfect matchings of $G$. In other words, the matching generating function is a generalization of the number of perfect matchings.

Let $G$ be weighted (reflectively) symmetric graph that is also bipartite. Without loss of generality, we always assume in this paper that the symmetry axis $\ell$ of $G$ is horizontal. Assume that the vertices of $G$ lying on $\ell$ form a cut set (i.e. the removal of those vertices separates $G$ into two disconnected parts). We say $\ell$ separates $G$. Color the vertices in two vertex classes of the bipartite graph $G$ black and white, so that the leftmost vertex of $G$ lying on $\ell$ is black. We define two subgraphs $G^{+}$and $G^{-}$of $G$ as follows.

It is easy to see that if $G$ has perfect matchings, then $G$ must have an even number of vertices on the symmetry $\ell$ (otherwise, the numbers of vertices of two vertex classes of $G$ are different, and $G$ does not have any perfect matchings). Therefore, we assume that the number of vertices of $G$ lying on $\ell$ is $2 w(G)$, for some integer $w(G)(w(G)$ was called the width of $G$ in [1]). Assume $a_{1}, b_{1}, a_{2}, b_{2}, \ldots, a_{w(G)}, b_{w(G)}$ are the vertices of $G$ lying on $\ell$ as they occur from left to right. Going along the line $\ell$ from left to right, we delete the edges of $G$ that touch white $a_{i}$ 's and black $b_{i}$ 's from above, and delete the edges of $G$ that touch black $a_{i}$ 's and white $b_{i}$ 's from below (see Figure 5 for an example). We notice that this process yields deletions of the same kind at the endpoints of each edge 
lying on $\ell$. We reduce the weights of those edges by half, and leave the weights of other edges unchanged. Since $\ell$ separates $G$, the resulting graph is disconnected into two parts. Denote by $G^{+}$and $G^{-}$the part above and below $\ell$, respectively.

Theorem 6 (Factorization Theorem, Ciucu [1]). Let $G$ be a bipartite weighted symmetric graph separated by its symmetry axis. Then

$$
\mathrm{M}(G)=2^{w(G)} \mathrm{M}\left(G^{+}\right) \mathrm{M}\left(G^{-}\right) .
$$

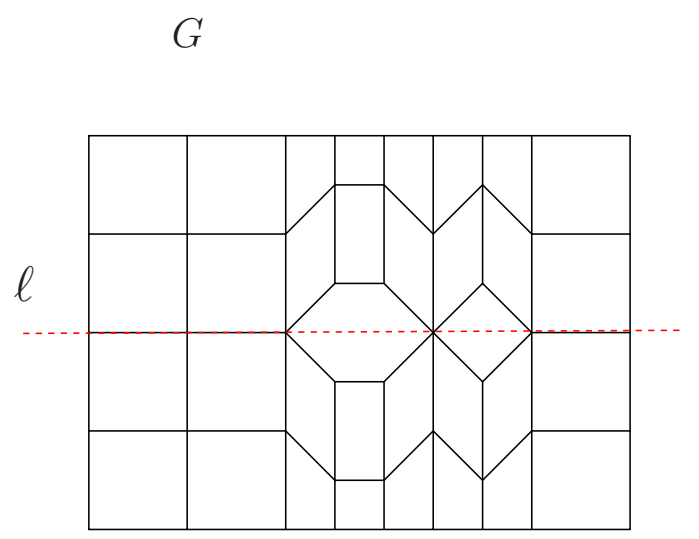

(a)

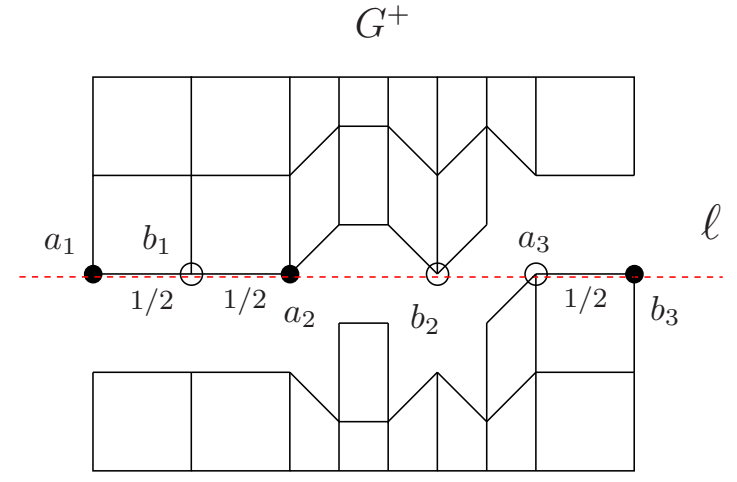

$G^{-}$

(b)

Figure 5: A symmetric graph $G$ (a), and two graphs $G^{+}$and $G^{-}$after the deleting process (b).

By applying the Factorization Theorem, we get new properties of quartered Aztec diamonds as follows.

Lemma 7. For $n \geqslant 1$

$$
\begin{gathered}
\mathrm{M}\left(A R_{2 n, 4 n}\left(\mathcal{B}_{n}\right)\right)=2^{n} \mathrm{~T}(R(4 n)) \mathrm{T}\left(K_{a}(4 n)\right), \\
\mathrm{M}\left(A R_{2 n, 4 n}\left(\mathcal{A}_{n}\right)\right)=2^{n} \mathrm{~T}(R(4 n)) \mathrm{T}\left(K_{n a}(4 n)\right), \\
\mathrm{M}\left(\overline{A R}_{2 n, 4 n-1}\left(\mathcal{A}_{n}\right)\right)=2^{n} \mathrm{~T}(R(4 n-1)) \mathrm{T}\left(K_{a}(4 n-1)\right), \\
\mathrm{M}\left(\overline{A R}_{2 n, 4 n-1}\left(\mathcal{B}_{n}\right)\right)=2^{n} \mathrm{~T}(R(4 n-1)) \mathrm{T}\left(K_{n a}(4 n-1)\right),
\end{gathered}
$$

where $\mathcal{A}_{n}=\{1,3, \ldots, 2 n-1\} \cup\{2 n+2,2 n+4, \ldots, 4 n\}$ and where $\mathcal{B}_{n}=\{2,4, \ldots, 2 n\} \cup\{2 n+1,2 n+3, \ldots, 4 n-1\}$. 


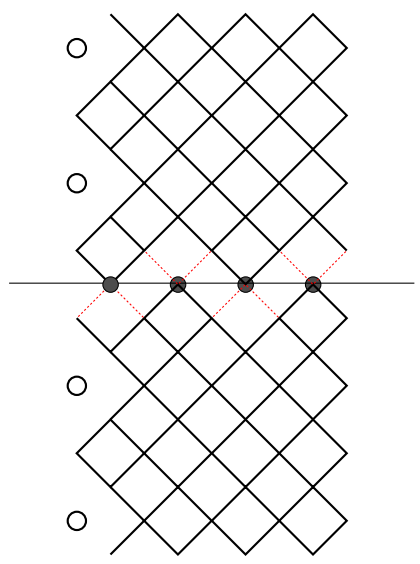

(a)

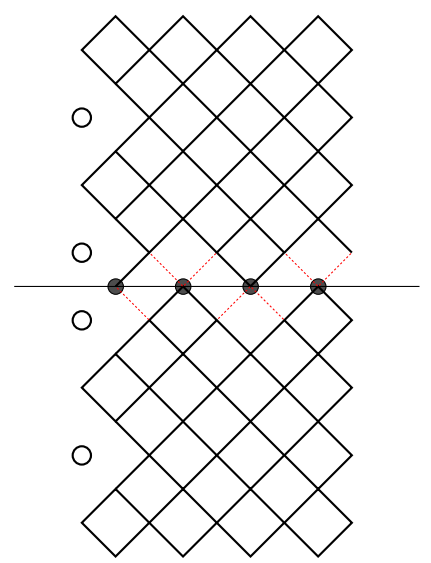

(b)

Figure 6: Illustrating the proofs of the equalities (18) and (19) in Lemma 7. The white circles indicate the vertices removed in the holey Aztec rectangle, and the red dotted edges indicate the edges deleted.

Proof. Apply the Factorization Theorem to the graph $G$ obtained from $A R_{2 n, 4 n}\left(\mathcal{B}_{n}\right)$ by rotating it $90^{\circ}$ clockwise. $G$ has a horizontal symmetry axis $\ell$. There are $2 n$ vertices of $G$ on $\ell$, so $w(G)=n$. We notice that in this case all vertices of $G$ on $\ell$ are black, and there are no edges of $G$ lying on $\ell$. It is easy to see that $G^{+}$is isomorphic to the dual graph of $K_{a}(4 n)$, and $G^{-}$is isomorphic to the dual graph of $R(4 n)$ (see Figure 6(a) for the case $n=2$ ). Then by Factorization Theorem, we get

$$
\begin{aligned}
\mathrm{M}\left(A R_{2 n, 4 n}\left(\mathcal{B}_{n}\right)\right) & =\mathrm{M}(G) \\
& =2^{w(G)} \mathrm{M}\left(G^{+}\right) \mathrm{M}\left(G^{-}\right) \\
& =2^{n} \mathrm{M}\left(K_{a}(4 n)\right) \mathrm{M}(R(4 n)) .
\end{aligned}
$$

This implies (18).

Again, we apply the Factorization Theorem to the graph $\bar{G}$ obtained from $A R_{2 n, 4 n}\left(\mathcal{A}_{n}\right)$ by rotating $90^{\circ}$ clockwise. $\bar{G}$ has also a horizontal symmetry axis $\ell^{\prime}$. It is easy to see that $\bar{G}^{+}$is isomorphic to the dual graph of $R(4 n)$, and $\bar{G}^{-}$is isomorphic to the dual graph of $K_{n a}(4 n)$ (the case $n=2$ is illustrated in Figure 6(b)). Moreover, it is easy to see $w(\bar{G})=n$, all vertices of $\bar{G}$ on $\ell^{\prime}$ are black, and there are no edges of $\bar{G}$ lying on $\ell^{\prime}$. Thus, we obtain

$$
\begin{aligned}
\mathrm{M}\left(\overline{A R}_{2 n, 4 n}\left(\mathcal{A}_{n}\right)\right) & =\mathrm{M}(\bar{G}) \\
& =2^{w(\bar{G})} \mathrm{M}\left(\bar{G}^{+}\right) \mathrm{M}\left(\bar{G}^{-}\right) \\
& =2^{n} \mathrm{M}(R(4 n)) \mathrm{M}\left(K_{n a}(4 n)\right),
\end{aligned}
$$




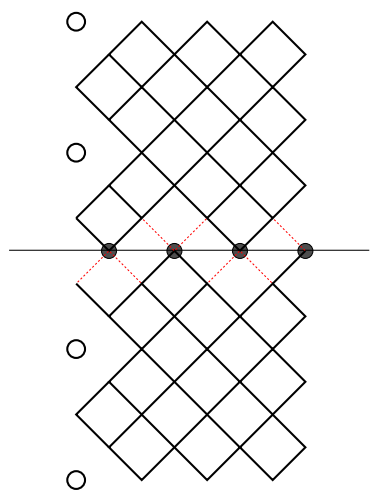

(a)

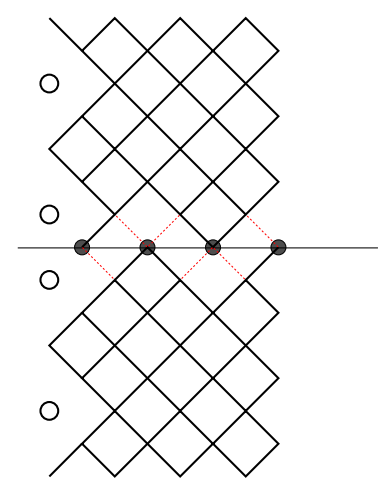

(b)

Figure 7: Illustrating the proofs of the equalities (20) and (21) in Lemma 7. The white circles indicate the vertices removed in the holey Aztec rectangle, and the red dotted edges indicate the edges deleted.

which implies (19).

Similarly, two equalities (20) and (21) can be obtained from applying the Factorization Theorem to the graphs $\overline{A R}_{2 n, 4 n-1}\left(\mathcal{A}_{n}\right)$ and $\overline{A R}_{2 n, 4 n-1}\left(\mathcal{B}_{n}\right)$ (after rotated $90^{0}$ clockwise). The proofs of the two equalities are illustrated in Figures 7(a) and (b), respectively. In particular, in Figure 7(a), the part above the symmetry axis is isomorphic to the dual graph of $K_{a}(4 n-1)$ and the part below the symmetry axis is isomorphic to the dual graph of $R(4 n-1)$, for $n=2$. In Figure 7(b), the part above the symmetry axis is isomorphic to the dual graph of $R(4 n-1)$ and the part below the symmetry axis is isomorphic to the dual graph of $K_{n a}(4 n-1)$, for $n=2$.

Let $S=\left\{s_{1}, s_{2}, \ldots, s_{t}\right\}$ be a nonempty finite set of real numbers, we define the operator $\Delta$ by

$$
\Delta(S):=\prod_{1 \leqslant i<j \leqslant t}\left(s_{j}-s_{i}\right) .
$$

Lemma 8. Let $\mathcal{A}_{n}$ and $\mathcal{B}_{n}$ be two sets defined in Lemma 7. For any $n \geqslant 1$

$$
\frac{\Delta\left(\mathcal{A}_{n}\right)}{\Delta\left(\mathcal{B}_{n}\right)}=\prod_{1 \leqslant i, j \leqslant n} \frac{2 n+1+2 j-2 i}{2 n-1+2 j-2 i} .
$$

Proof. We can partition $\mathcal{A}_{n}=\mathcal{C}_{n} \sqcup \mathcal{D}_{n}$, where $\mathcal{C}_{n}=\{1,3, \ldots, 2 n-1\}$ and where 
$\mathcal{D}_{n}=\{2 n+2,2 n+4, \ldots, 4 n\}$. Therefore

$$
\begin{aligned}
\Delta\left(\mathcal{A}_{n}\right)= & \prod_{i<j \in \mathcal{C}_{n}}(j-i) \prod_{i<j \in \mathcal{D}_{n}}(j-i) \prod_{i \in \mathcal{C}_{n}, j \in \mathcal{D}_{n}}(j-i) \\
= & \prod_{1 \leqslant i<j \leqslant n}((2 j-1)-(2 i-1)) \prod_{1 \leqslant i<j \leqslant n}((2 j+2 n)-(2 i+2 n)) \\
& \times \prod_{1 \leqslant i, j \leqslant n}((2 j+2 n)-(2 i-1)) \\
= & \prod_{1 \leqslant i<j \leqslant n} 2(j-i) \prod_{1 \leqslant i<j \leqslant n} 2(j-i) \prod_{1 \leqslant i, j \leqslant n}(2 n+1+2 j-2 i) \\
= & 2^{n(n-1)}\left(\prod_{1 \leqslant i<j \leqslant n}(j-i)\right)^{2} \prod_{1 \leqslant i, j \leqslant n}(2 n+1+2 j-2 i) .
\end{aligned}
$$

Similarly, we have a partition of $\mathcal{B}_{n}=\mathcal{C}_{n}^{\prime} \sqcup \mathcal{D}_{n}^{\prime}$, where $\mathcal{C}_{n}^{\prime}=\{2,4, \ldots, 2 n\}$ and $\mathcal{D}_{n}^{\prime}=$ $\{2 n+1,2 n+3, \ldots, 4 n-1\}$. We also get

$$
\begin{aligned}
\Delta\left(\mathcal{B}_{n}\right)= & \prod_{i<j \in \mathcal{C}_{n}^{\prime}}(j-i) \prod_{i<j \in \mathcal{D}_{n}^{\prime}}(j-i) \prod_{i \in \mathcal{C}_{n}^{\prime}, j \in \mathcal{D}_{n}^{\prime}}(j-i) \\
= & \prod_{1 \leqslant i<j \leqslant n}((2 j)-(2 i)) \prod_{1 \leqslant i<j \leqslant n}((2 j-1+2 n)-(2 i-1+2 n)) \\
& \times \prod_{1 \leqslant i, j \leqslant n}((2 j-1+2 n)-2 i) \\
= & \prod_{1 \leqslant i<j \leqslant n} 2(j-i) \prod_{1 \leqslant i<j \leqslant n} 2(j-i) \prod_{1 \leqslant i, j \leqslant n}(2 n-1+2 j-2 i) \\
= & 2^{n(n-1)}\left(\prod_{1 \leqslant i<j \leqslant n}(j-i)\right)^{2} \prod_{1 \leqslant i, j \leqslant n}(2 n-1+2 j-2 i) .
\end{aligned}
$$

Then the equality (24) follows.

We are now ready to prove Theorem 1.

Proof of Theorem 1. Since the dual graph $G$ of $R(n)$ is a bipartite graph, the numbers of vertices in two vertex classes of $G$ must be the same if $G$ admits perfect matchings. Let us color the vertices in the two vertex classes of $G$ black and white. For definiteness, we always color the vertex on the northwestern side of the dual graph of $R(n)$ white (see Figures $8(\mathrm{a})$ and (b) for the colorings of $R(4 n+1)$ and $R(4 n+2)$, for $n=2$, respectively).

By enumerating particularly, the dual graph of $R(4 n+1)$ has $(2 n+1)^{2}-(n+1)$ white vertices and $(2 n+1)^{2}-n$ black vertices; the dual graph of $R(4 n+2)$ has $(2 n+1)(2 n+2)-n$ white vertices and $(2 n+1)(2 n+2)-(n+1)$ black vertices. It means that the numbers of vertices in two vertex classes of $R(4 n+1)$ (resp., of $R(4 n+2)$ ) are not the same, 


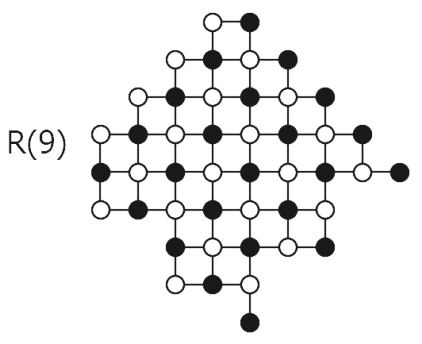

(a)

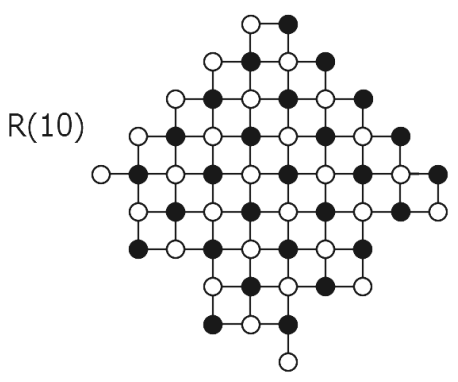

(b)

Figure 8: The dual graphs of $R(9)$ and $R(10)$ with two vertex classes (black and white).

so $R(4 n+1)$ (resp., $R(4 n+2))$ does not have any perfect matchings. This implies the equality (1).

Next, we prove five equalities (2)-(6) by induction on $n \geqslant 1$.

It is easy to verify those formulas for $n=1$. Assume that the formulas hold for some $n \geqslant 1$, we will show that they hold also for $n+1$.

We have from Lemmas 2 and 3, and induction hypothesis

$$
\begin{array}{rlrl}
\mathrm{T}\left(K_{a}(4 n+4)\right) & =\mathrm{T}\left(K_{a}(4 n+2)\right) & (\text { by Eq. } & (11)) \\
& =2^{n+1} \mathrm{~T}\left(K_{n a}(4 n+1)\right) & (\text { by Eq. (10)) } \\
& =2^{2 n+1} \mathrm{~T}\left(K_{n a}(4 n)\right) & (\text { by Eq. (8)) } \\
& =2^{2 n+1} 2^{n(3 n-1) / 2} \prod_{1 \leqslant i \leqslant j \leqslant n} \frac{2 i+2 j-1}{i+j-1} \quad(\text { by Eq. (5) for } n) \\
& =2^{\left(3 n^{2}+3 n+2\right) / 2} \prod_{1 \leqslant i \leqslant j \leqslant n}\left(\frac{i+(j+1)-1}{i+j-1} \cdot \frac{2 i+2(j+1)-3}{i+(j+1)-1}\right) \\
& =2^{(n+1)(3 n+2) / 2} 2^{-n} \prod_{1 \leqslant i \leqslant j \leqslant n} \frac{i+j}{i+j-1} \prod_{1 \leqslant i<j \leqslant n+1} \frac{2 i+2 j-3}{i+j-1} \\
& =2^{(n+1)(3(n+1)-1) / 2} 2^{-n} \prod_{j=1}^{n} \frac{2 j}{j} \prod_{1 \leqslant i<j \leqslant n+1} \frac{2 i+2 j-3}{i+j-1} \\
& =2^{(n+1)(3(n+1)-1) / 2} \prod_{1 \leqslant i<j \leqslant n+1} \frac{2 i+2 j-3}{i+j-1} .
\end{array}
$$

It means that (3) holds for $n+1$. 
By Lemmas 5, 7 and 8, and the induction hypothesis (equality (5) for $n$ ), we obtain

$$
\begin{aligned}
& \mathrm{T}\left(K_{a}(4 n+5)\right)=\mathrm{T}\left(K_{a}(4 n+3)\right) \\
& =\mathrm{T}\left(K_{n a}(4 n+3)\right) \frac{\mathrm{T}\left(K_{a}(4 n+3)\right)}{\mathrm{T}\left(K_{n a}(4 n+3)\right)} \\
& =\mathrm{T}\left(K_{n a}(4 n+1)\right) \frac{\mathrm{T}\left(K_{a}(4 n+3)\right)}{\mathrm{T}\left(K_{n a}(4 n+3)\right)} \quad \text { (by Eq. (14)) } \\
& =2^{n} \mathrm{~T}\left(K_{n a}(4 n)\right) \frac{\mathrm{T}\left(K_{a}(4 n+3)\right)}{\mathrm{T}\left(K_{n a}(4 n+3)\right)} \quad \text { (by Eq. (8)) } \\
& =2^{n} \mathrm{~T}\left(K_{n a}(4 n)\right) \frac{\mathrm{M}\left(\overline{A R}_{2 n+2,4 n+3}\left(\mathcal{A}_{n+1}\right)\right)}{\mathrm{M}\left(\overline{A R}_{2 n+2,4 n+3}\left(\mathcal{B}_{n+1}\right)\right)} \quad \text { (by Eqs. (20) and (21)) } \\
& \left.=2^{n} \mathrm{~T}\left(K_{n a}(4 n)\right) \frac{\Delta\left(\mathcal{A}_{n+1}\right)}{\Delta\left(\mathcal{B}_{n+1}\right)} \quad \text { (by Lemma } 5\right) \\
& =2^{n} \mathrm{~T}\left(K_{n a}(4 n)\right) \prod_{1 \leqslant i, j \leqslant n+1} \frac{2 n+3+2 j-2 i}{2 n+1+2 j-2 i} \quad \text { (by Lemma 8) } \\
& =2^{n}\left(2^{n(3 n-1) / 2} \prod_{1 \leqslant i \leqslant j \leqslant n} \frac{2 i+2 j-1}{i+j-1}\right) \prod_{1 \leqslant i, j \leqslant n+1} \frac{2 n+3+2 j-2 i}{2 n+1+2 j-2 i} \\
& =2^{n}\left(2^{n(3 n-1) / 2} \frac{\prod_{1 \leqslant i \leqslant j \leqslant n+1} \frac{2 i+2 j-1}{i+j-1}}{\prod_{1 \leqslant i \leqslant n+1} \frac{2 i+2 n+1}{i+n}}\right) \prod_{1 \leqslant j \leqslant n+1} \frac{2 n+1+2 j}{2 j-1} \\
& =2^{n}\left(2^{n(3 n-1) / 2} \prod_{1 \leqslant i \leqslant j \leqslant n+1} \frac{2 i+2 j-1}{i+j-1}\right) \prod_{1 \leqslant j \leqslant n+1} \frac{j+n}{2 j-1} \\
& =2^{n}\left(2^{n(3 n-1) / 2} \prod_{1 \leqslant i \leqslant j \leqslant n+1} \frac{2 i+2 j-1}{i+j-1}\right) \frac{(2 n+1) ! / n !}{(2 n+1) ! /\left(2^{n} n !\right)} \\
& =2^{(n+1)(3(n+1)-3) / 2} \prod_{1 \leqslant i \leqslant j \leqslant n+1} \frac{2 i+2 j-1}{i+j-1} \text {. }
\end{aligned}
$$

This implies that (4) holds for $n+1$.

We verify next the equality (5) for $n+1$.

$$
\begin{aligned}
\mathrm{T}\left(K_{n a}(4 n+6)\right) & =\mathrm{T}\left(K_{n a}(4 n+4)\right) \\
& =2^{n+1} \mathrm{~T}\left(K_{a}(4 n+3)\right) \\
& =2^{n+1}\left(2^{(n+1)(3(n+1)-3) / 2} \prod_{1 \leqslant i \leqslant j \leqslant n+1} \frac{2 i+2 j-1}{i+j-1}\right) \quad(\text { by Eq. (13)) } \\
(\text { by Eq. (9) }) & (51) \\
& =2^{(n+1)(3(n+1)-1) / 2} \prod_{1 \leqslant i \leqslant j \leqslant n+1} \frac{2 i+2 j-1}{i+j-1}
\end{aligned}
$$


Similarly, we can get the equality (6) for $n+1$, as follows:

$$
\begin{array}{rlr}
\mathrm{T}\left(K_{n a}(4 n+3)\right)= & \mathrm{T}\left(K_{n a}(4 n+1)\right) & (\text { by Eq. (14)) } \\
& =2^{-(n+1)} \mathrm{T}\left(K_{a}(4 n+2)\right) & (\text { by Eq. (10)) } \\
& =2^{-(n+1)}\left(2^{(n+1)(3(n+1)-1) / 2} \prod_{1 \leqslant i<j \leqslant n+1} \frac{2 i+2 j-3}{i+j-1}\right)
\end{array}
$$

(by Eq. (3) for $n+1$ )

$$
=2^{(n+1)(3(n+1)-3) / 2} \prod_{1 \leqslant i<j \leqslant n+1} \frac{2 i+2 j-3}{i+j-1} .
$$

Finally, we verify the equality (2) for $n+1$.

Denote by $[n]:=\{1,2,3, \ldots, n\}$ the set of the first $n$ positive integers. Similar to the proof of Lemma 8, we partition $[2 n+2]=\mathcal{E}_{n} \sqcup \mathcal{F}_{n}$, where $\mathcal{E}_{n}=[n+1]$ and $\mathcal{F}_{n}=$ $\{n+2, n+3, \ldots, 2 n+2\}$, and obtain

$$
\begin{aligned}
\Delta([2 n+2])= & \prod_{i<j \in \mathcal{E}_{n}}(j-i) \prod_{i<j \in \mathcal{F}_{n}}(j-i) \prod_{i \in \mathcal{E}_{n}, j \in \mathcal{F}_{n}}(j-i) \\
= & \prod_{1 \leqslant i<j \leqslant n+1}(j-i) \prod_{1 \leqslant i<j \leqslant n+1}((n+1+j)-(n+1+i)) \\
& \times \prod_{1 \leqslant i, j \leqslant n+1}((n+1+j)-i) \\
= & \left(\prod_{1 \leqslant i<j \leqslant n+1}(j-i)\right)^{2} \prod_{1 \leqslant i, j \leqslant n+1}(n+1+j-i) .
\end{aligned}
$$

Thus, by Lemmas 4 and 8, we get

$$
\begin{aligned}
\mathrm{M}\left(A R_{2 n+2,4 n+4}\left(\mathcal{A}_{n+1}\right)\right)=2^{(2 n+2)(2 n+3) / 2} \frac{\Delta\left(\mathcal{A}_{n+1}\right)}{\Delta([2 n+2])} & \\
= & 2^{(2 n+2)(2 n+3) / 2} \\
& \times \frac{2^{(n+1) n}\left(\prod_{1 \leqslant i<j \leqslant n+1}(j-i)\right)^{2} \prod_{1 \leqslant i, j \leqslant n+1}(2 n+3+2 j-2 i)}{\left(\prod_{1 \leqslant i<j \leqslant n+1}(j-i)\right)^{2} \prod_{1 \leqslant i, j \leqslant n+1}(n+1+j-i)} \\
= & 2^{(2 n+2)(2 n+3) / 2} 2^{(n+1) n} \prod_{1 \leqslant i, j \leqslant n+1} \frac{2 n+3+2 j-2 i}{n+1+j-i} \\
= & 2^{(2 n+2)(2 n+3) / 2} 2^{(n+1) n} \prod_{1 \leqslant t, j \leqslant n+1} \frac{2 t+2 j-1}{t+j-1} \quad(\text { let } t:=n+1-i) \\
= & \left.2^{(n+1)(3 n+3)} \prod_{1 \leqslant i, j \leqslant n+1} \frac{2 i+2 j-1}{i+j-1} \quad \text { (let } i:=t\right) .
\end{aligned}
$$


By (7) and (19), together with the above formula for $\mathrm{M}\left(A R_{2 n+2,4 n+4}\left(\mathcal{A}_{n+1}\right)\right)$, we get

$$
\begin{aligned}
& 2^{n+1} \mathrm{~T}(R(4 n+3))=\mathrm{T}(R(4 n+4)) \quad \text { (by Eq. (7)) } \\
& =2^{-(n+1)} \frac{\mathrm{M}\left(A R_{2 n+2,4 n+4}\left(\mathcal{A}_{n+1}\right)\right)}{\mathrm{T}\left(K_{n a}(4 n+4)\right)} \quad \text { (by Eq. (19)) } \\
& \left.=2^{-(n+1)} \frac{2^{(n+1)(3 n+3)} \prod_{1 \leqslant i, j \leqslant n+1} \frac{2 i+2 j-1}{i+j-1}}{2^{(n+1)(3(n+1)-1) / 2} \prod_{1 \leqslant i \leqslant j \leqslant n+1} \frac{2 i+2 j-1}{i+j-1}} \quad \text { (by Eq. (5) for } n+1\right) \\
& =2^{(n+1)(3(n+1)-1) / 2} \prod_{1 \leqslant j<i \leqslant n+1} \frac{2 i+2 j-1}{i+j-1} \\
& =2^{(n+1)(3(n+1)-1) / 2} \prod_{1 \leqslant i<j \leqslant n+1} \frac{2 i+2 j-1}{i+j-1} \quad \text { (let } i:=j \text { and } j:=i \text {. }
\end{aligned}
$$

This verifies that the equality (2) holds for $n+1$, and the theorem follows from induction principle.

\section{Acknowledgements}

Thanks to Professor Mihai Ciucu and Professor James Propp for giving me the manuscript of $[5]$.

\section{References}

[1] M. Ciucu. Enumeration of perfect matchings in graphs with reflective symmetry. $J$. Combin. Theory Ser. A 77: 67-97, 1997.

[2] M. Ciucu. A complementation theorem for perfect matchings of graphs having a cellular completion. J. Combin. Theory Ser. A 81: 34-68, 1998.

[3] N. Elkies, G. Kuperberg, M. Larsen, and J. Propp. Alternating-sign matrices and domino tilings (Part I). J. Algeberaic Combin. 1: 111-132, 1992.

[4] H. Helfgott and I. M. Gessel. Enumeration of tilings of diamonds and hexagons with defects. Electron. J. Combin. 6: R16, 1999.

[5] W. Jockusch and J. Propp. Antisymmetric monotone triangles and domino tilings of quartered Aztec diamonds. Unpublished work.

[6] C. Krattenthaler. Schur function identities and the number of perfect matchings of holey Aztec rectangles. q-series from a contemporary perspective (South Hadley, MA): 335-349, 1998. Contemp. Math., 254, Amer. Math. Soc., Providence, RI, 2000. 